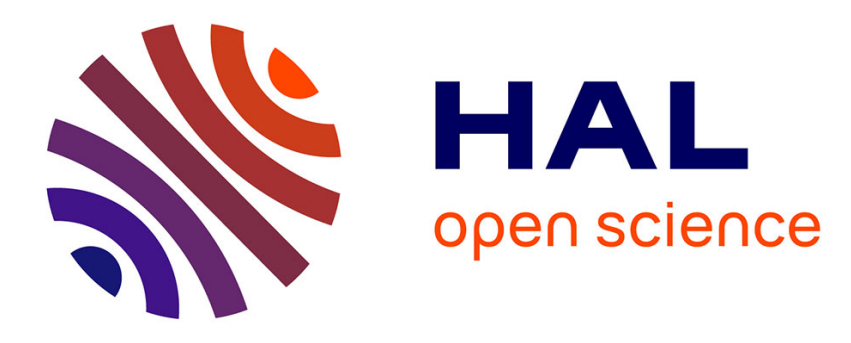

\title{
Les fonctions des connaissances produites par les sciences de gestion \\ Matthieu Mandard
}

\section{To cite this version:}

Matthieu Mandard. Les fonctions des connaissances produites par les sciences de gestion. Question(s) de Management, 2020, 2 (32), pp.157-165. 10.3917/qdm.212.0157 . halshs-02961525

\section{HAL Id: halshs-02961525 \\ https://shs.hal.science/halshs-02961525}

Submitted on 15 Apr 2021

HAL is a multi-disciplinary open access archive for the deposit and dissemination of scientific research documents, whether they are published or not. The documents may come from teaching and research institutions in France or abroad, or from public or private research centers.
L'archive ouverte pluridisciplinaire HAL, est destinée au dépôt et à la diffusion de documents scientifiques de niveau recherche, publiés ou non, émanant des établissements d'enseignement et de recherche français ou étrangers, des laboratoires publics ou privés. 


\title{
Les fonctions des connaissances produites par les sciences de gestion ${ }^{1}$
}

\author{
Matthieu MANDARD \\ Maître de conférences en sciences de gestion \\ Université Rennes 1 \\ Centre de Recherche en Économie et Management (CREM - UMR CNRS 6211) \\ 11 rue Jean Macé - CS 70803 - 35708 RENNES Cedex 7 - FRANCE \\ E-mail : matthieu.mandard@univ-rennes1.fr
}




\section{Les fonctions des connaissances produites par les sciences de gestion}

Résumé : À quoi peuvent servir les connaissances que nous produisons, nous chercheurs en sciences de gestion? Si la question a souvent été traitée, elle n'a jusqu'à présent pas fait l'objet

d'une réponse exhaustive : c'est ce que nous proposons de faire dans cet article. À partir d'un examen de la littérature, nous proposons une typologie qui nous permet de mettre en évidence quatre principales fonctions : d'ordre épistémique, pratique, économique et idéologique. Nous présentons ensuite leurs caractéristiques. Nous discutons finalement les implications de ce travail, en termes de formation à la recherche comme en matière de pilotage des activités scientifiques.

Mots clés : connaissances ; fonctions ; recherche scientifique ; sciences de gestion

\section{The functions of the knowledge produced in management science}

Summary: What can be the uses of the knowledge that we produce, we scholars in management science? While the question has often been examined, to date, it has not received an exhaustive answer: this is what we propose to do in this paper. Based on an examination of the literature, we propose a typology that allows us to identify four main functions: epistemic, practical, economic, and ideological. We then present the characteristics of each of them. We finally discuss the implications of the article, both for research training and for the governance of scientific activities.

Keywords: functions; knowledge; management science; scientific research 


\section{INTRODUCTION}

À quoi peuvent servir les connaissances que nous produisons, nous chercheurs en sciences de gestion? Il s'agit là d'une interrogation récurrente dans notre discipline, et à laquelle sont souvent apportées des réponses telles que : développer notre compréhension des phénomènes organisationnels, étoffer nos enseignements, ou améliorer les pratiques managériales (e.g., David, Hatchuel et Laufer, 2012 ; Pichault, Lisein, Rondeaux et Xhauflair, 2008 ; Carton et Mouricou, 2017 ; Verstraete, 2007). Est-ce là la totalité des usages possibles ? Il y en a-t-il d'autres ? Et comment les catégoriser?

L'objectif de cet article est de répondre à ces questions. Plus précisément, il s'agit ici de montrer que les fonctions des connaissances produites par les sciences de gestion sont au nombre de quatre - d'ordre épistémique, pratique, économique et idéologique -, puis de présenter les caractéristiques de chacune d'elles². Si la plupart de ces fonctions sont déjà bien connues, il s'agit de les recenser, de montrer leur articulation, et d'insister sur l'une d'entre elles, la fonction idéologique, qui est souvent passée sous silence.

De notre point de vue, l'intérêt de ce travail à vocation didactique est double. Il nous semble qu'un tel article pourrait être utile en matière de formation à la recherche, qu'il s'agisse de préparer de futurs collègues à leurs activités de chercheurs ou d'informer toute personne intéressée par les enjeux de la recherche scientifique. Il nous semble que ce travail pourrait aussi être utile en matière de pilotage des activités de recherche, en ce qu'il permet de rappeler les tensions associées à chacune des fonctions présentées.

L'article est structuré de la manière suivante. Nous présentons tout d'abord la typologie qui nous permet de mettre en évidence les quatre principales fonctions des connaissances produites par les sciences de gestion. Nous détaillons ensuite les caractéristiques de chacune de ces fonctions. Nous terminons par une présentation des implications de l'article.

$2 \quad$ Nous tenons d'emblée à souligner le fait que cet article ne porte pas sur les objectifs susceptibles d'être poursuivis par les sciences de gestion, tels que l'accroissement de la performance économique ou l'amélioration du bien-être social. Il s'agit ici de recenser les diverses fonctions des connaissances que nous produisons, lesquelles peuvent servir différents objectifs tels que ceux que nous venons d'évoquer (sur ce sujet, voir Mandard, 2020). 


\section{La typologie proposée}

Notre recension des fonctions des connaissances produites par les sciences de gestion est fondée sur un cadre conceptuel original que nous avons bâti. Nous en présentons tout d'abord les principes d'élaboration, puis le cadre lui-même.

\subsection{Principes d'élaboration de la typologie}

Afin d'élaborer cette typologie, nous avons examiné la littérature traitant des fonctions des connaissances produites par les sciences de gestion. Il s'est agit pour l'essentiel d'articles francophones et anglophones consacrés à l'épistémologie des sciences de gestion, que nous avons parcourus de proche en proche afin de cerner les fonctions des connaissances habituellement évoquées. Nous avons estimé cette revue de littérature terminée lorsque toute lecture complémentaire n'apportait plus de propos nouveaux relativement à notre problématique, suivant un principe de saturation théorique (Glaser et Strauss, 1967).

Nous avons ensuite tenté d'identifier l'appareillage conceptuel le mieux à même de rendre compte de ces fonctions. Nous avons pour ce faire procédé par aller et retour avec la littérature. Nous avons jugé notre proposition théorique satisfaisante dès lors qu'elle nous a paru correctement rendre compte de la diversité des fonctions connues (Doty et Glick, 1994). Il nous semble en effet qu'il n'existe pas de fonctions des connaissances produites par les sciences de gestion autres que celles que nous détaillons dans notre typologie.

\subsection{Présentation de la typologie}

La typologie que nous avons mise au point retient quatre principales fonctions des connaissances produites par les sciences de gestion : d'ordre épistémique, pratique, économique et idéologique. Cette typologie est bâtie autour de deux dimensions (Tableau 1). 
Tableau 1 : Les fonctions des connaissances produites par les sciences de gestion : une proposition de typologie ${ }^{3}$

Intérêt porté à la connaissance

$\begin{array}{cc}\text { Intrinsèque à la } & \text { Extrinsèque à la } \\ \text { connaissance produite } & \text { connaissance produite }\end{array}$

\section{La connaissance}

Manière dont la

scientifique constitue Pratique Économique

connaissance est

un moyen

employée

\section{La connaissance}

scientifique représente Épistémique Idéologique

une finalité

La première dimension à laquelle nous aurons recours relève de l'intérêt porté à la connaissance produite. Nous reprendrons ici la distinction populaire entre motivation intrinsèque et motivation extrinsèque, largement utilisée dans le champ du comportement organisationnel (Ryan et Deci, 2000). Les fonctions pratiques et épistémiques relèvent d'un intérêt intrinsèque dans les connaissances produites, dans la mesure où ce sont des connaissances bien particulières qui font l'objet d'un intérêt managérial ou théorique, et pas d'autres. Les fonctions économiques et idéologiques relèvent quant à elles d'un intérêt extrinsèque, car ce qui est en jeu, ce sont d'abord et avant tout les transactions que les connaissances produites permettent de réaliser ou les idées qu'elles permettent de véhiculer - et pas les connaissances en elles-mêmes ${ }^{4}$.

3 Cette typologie s’avère suivre un raisonnement analogue à une typologie que nous avons précédemment élaborée sur un tout autre sujet, celui des usages organisationnels des espaces géographiques (Mandard 2019). Par ailleurs, bien qu'élaborée à partir d'une analyse conduite au niveau des sciences de gestion, cette typologie est très probablement transposable à tout domaine de recherche. Quelle que soit la discipline envisagée, il nous semble en effet que toute connaissance produite puisse avoir une fonction épistémique, pratique, économique, ou idéologique.

4 À titre d'exemple, un même gain économique (par exemple, une prime à la publication d'article) peut en effet être réalisé au travers de n’importe quel article publié dans une revue bien classée. De la même manière, une même idéologie (par exemple, la valorisation de la performance économique) peut être véhiculée en conduisant des travaux en stratégie qui examinent la performance des entreprises sur un marché, ou en menant des travaux en gestion des ressources humaines consacrés à la performance des employés au sein d'une entreprise. 
La seconde dimension à laquelle nous aurons recours relève de la manière dont la connaissance produite est employée, avec ici une distinction moyen-fin. S'agissant des fonctions pratiques et économiques, les connaissances constituent des moyens de changer les pratiques de gestion ou d'acquérir diverses contreparties. Dans le cas des fonctions épistémiques et idéologiques, les connaissances permettent de mieux comprendre le monde ou véhiculent des idées dès lors qu'elles sont produites : elles constituent en elles-mêmes une finalité .

\section{Les caractéristiques des fonctions des connaissances produites par les sciences de gestion}

Nous présentons ici les caractéristiques - la définition, les principes essentiels de mise en œuvre ainsi que les limites - des quatre fonctions que nous avons identifiées précédemment, fonctions dont nous introduisons d'emblée la définition en Tableau 2.

Tableau 2 : Les fonctions des connaissances produites par les sciences de gestion

\section{Type de fonction Définition de la fonction}

Épistémique Les connaissances scientifiques offrent une compréhension du monde

Pratique Les connaissances scientifiques permettent une action sur le monde

Économique Les connaissances scientifiques représentent une contribution pour le monde

Idéologique Les connaissances scientifiques témoignent d'une vision du monde

\subsection{La fonction épistémique}

Première fonction que nous proposons de mettre en évidence, la fonction épistémique - du grec épistémè qui signifie savoir, connaissance - de la connaissance scientifique correspond à un intérêt porté à la connaissance pour elle-même. Les connaissances font l'objet de curiosité, leur acquisition est d'abord et avant tout motivée par le désir de comprendre et d'apprendre.

5 Bien entendu, une même production scientifique peut remplir plusieurs fonctions de manière simultanée. Ainsi, il est tout à fait possible de réaliser une recherche par intérêt épistémique tout en en retirant des bénéfices économiques (e.g., une prime ou une promotion). 
Il s'agit là d'un rapport à la connaissance mis en évidence dès la Grèce Antique, et notamment par Aristote, qui entame son œuvre La Métaphysique par l'affirmation suivante: "Tous les hommes désirent par nature savoir ». Cet intérêt pour la connaissance est plus tard évoqué par Saint Augustin, qui voit en la libido sciendi - le désir de savoir - l'une des passions qui s'imposent à l'homme. Il est aussi constitutif de la Renaissance, des Lumières, ou même de la société de la connaissance contemporaine (Jacques-Chaquin et Houdard, 1998). Il convient de noter que, s'il existe une tendance anthropologique à la curiosité en général, et à la curiosité scientifique en particulier, celle-ci est bien entendu variable en fonction des individus : chacun dispose en effet de centres d'intérêt particuliers (Loewenstein, 1994).

Cet intérêt porté à la connaissance scientifique est largement partagé par les chercheurs, et constitue souvent un moteur dans la conduite de leurs travaux (Simon, 2001). Il s'avère d'ailleurs partie intégrante de la recherche fondamentale qui, par définition, n'a pour but que de produire des connaissances pour elles-mêmes, sans applications immédiates. La curiosité scientifique est aussi connue pour animer les enfants, les étudiants et, plus généralement, toute personne qui souhaite en savoir davantage sur le fonctionnement du monde qui l'entoure (Loewenstein, 1994).

En sciences de gestion, si l'étude de la fréquence de cet usage reste à faire, l'intérêt pour les travaux conduits est souvent reconnu comme étant déterminant pour nombre de chercheurs. En 2006, une enquête menée au sein du comité de rédaction de l'Academy of Management Journal mettait ainsi en évidence l'importance donnée par les membres de ce comité au caractère intéressant des connaissances produites, dans la mesure où il constitue un vecteur d'émulation au sein de la communauté scientifique (Bartunek, Rynes et Ireland, 2006). Dans leur introduction au récent numéro de la Revue Française de Gestion intitulé « Recherche en gestion : retrouver du sens », les rédacteurs en chef invités (Moriceau, Laroche et Jardat, 2017) insistent eux aussi sur l'importance du goût pour le savoir qui les anime en tant que chercheurs - même s'ils déplorent le fait que la focalisation actuelle sur la publication dans des revues classées et la normalisation académique qui l'accompagne tend à brider cet intérêt au sein de notre communauté. De la même manière, l'intérêt des étudiants en gestion pour les connaissances qu'ils acquièrent est souvent rapporté. C'est notamment le cas des doctorants qui, ainsi que le mentionne Aggeri (2016) sont 
souvent désireux d'enrichir leurs connaissances et de développer leur réflexion au travers de la découverte de textes marquants.

S'il est populaire, le simple usage épistémique des connaissances produites en sciences de gestion ne va cependant pas sans rencontrer quelques critiques dans une discipline à vocation praxéologique. Il ne suffit pas d'affiner la compréhension des problématiques organisationnelles, il faut aussi employer les connaissances créées afin d'améliorer la performance économique c'est là un rôle historiquement assigné aux sciences de gestion - (Kieser, Nicolai et Seidl, 2015), afin de favoriser le progrès social - objectif lui aussi présent dès la naissance des sciences de gestion, mais popularisé seulement à partir du milieu du 20ème siècle avec l'apparition du concept de responsabilité sociale des entreprises - (Acquier et Aggeri, 2015), ou afin de participer à la réduction des impacts environnementaux des activités des organisations problématique qui s'impose peu à peu dans notre discipline (Berchicci et King, 2007). En d'autres termes, les connaissances que nous produisons doivent aussi avoir une fonction pratique, que nous examinons à présent.

\subsection{La fonction pratique}

Seconde fonction considérée, la fonction pratique correspond à un intérêt porté aux applications des connaissances produites. Contrairement à la fonction épistémique présentée précédemment, ici, les connaissances ne représentent pas une finalité : elles constituent un moyen d'améliorer les actions.

La conversion des connaissances produites en diverses applications semble avoir eu lieu de tout temps, puisque dès l'Antiquité des progrès sont ainsi réalisés en médecine, en architecture, ou en astronomie (Gingras, 2018). À partir du 19ème siècle, avec l'essor de la recherche académique, la recherche des implications pratiques des découvertes scientifiques se systématise, et la science devient très souvent un préalable aux progrès techniques.

Néanmoins, en sciences de gestion comme dans les autres sciences sociales, on assiste plutôt à la démarche inverse : les connaissances sont généralement précédées par les pratiques - ceci car les individus n'attendent pas les chercheurs pour agir. Le rôle historiquement assigné à notre 
discipline est donc celui d'étudier ces pratiques afin d'en améliorer la compréhension et la mise en œuvre (Pfeffer, 2007). C'est ainsi que la ligne d'assemblage, le laboratoire de recherche moderne, la structure divisionnelle, le toyotisme ou, plus récemment, le $\mathrm{CV}$ anonyme, les normes IFRS, les hedge funds ou le management environnemental sont des pratiques qui ont émergé avant de devenir des objets d'études destinées à les évaluer et à perfectionner leur déploiement (Birkinshaw, Hamel et Mol, 2008).

Il existe bien entendu des exceptions, puisque des outils tels que le tableau de bord prospectif, le modèle des 5 forces de la concurrence, le marketing mix ou le Modèle d'évaluation des actifs financiers (MEDAF) sont directement le fruit de réflexions théoriques. Pour prendre le cas de travaux francophones, le modèle C-K de pilotage de l'invention développé par Hatchuel et ses collègues de l'École des mines de Paris (Hatchuel, Weil et Le Masson, 2013), la méthodologie de gestion du changement inspirée de la sociologie de la traduction et élaborée par Pichault (2013), ou le management socio-économique théorisé par Savall et Zardet (2014) sont aussi des développements qui ont rencontré un certain écho en entreprise.

Bien qu'important - et traditionnellement requis au travers des habituelles préconisations managériales -, doit-on pour autant faire de cet usage pratique une condition nécessaire de toute production scientifique, et notamment en sciences de gestion? Il faut ici prendre garde à ce qu'une focalisation excessive sur cet usage ne conduise au renoncement de toute recherche sans application immédiate (Kieser et Leiner, 2009). Le risque serait alors grand d'observer un désintérêt pour des champs fondamentaux des sciences de gestion tels que la théorie des organisations, la finance comportementale, le comportement du consommateur ou le comportement organisationnel, dont la visée opérationnelle n'est pas immédiate mais incidente, et dont la compréhension est pourtant indispensable pour affiner les pratiques.

\subsection{La fonction économique}

Un usage économique peut également être fait des connaissances produites lorsque celles-ci représentent une contribution qui peut faire l'objet des contreparties les plus diverses - le terme économie renvoie donc ici aux problématiques d'allocation de ressources. Dans ce cas précis, 
l'enjeu n'est pas tant la nature des connaissances produites que ce que celles-ci permettent d'obtenir en retour: de même que pour la fonction pratique, les connaissances produites constituent donc ici aussi un moyen et non pas une finalité. Le travail scientifique est en effet une activité habituellement assortie de diverses rétributions.

Phénomène soutenu par le fort développement contemporain des techniques de bibliométrie, la production scientifique est un indicateur couramment utilisé pour la gestion de la carrière des chercheurs. En matière de recrutement tout d'abord, il s'agit d'un critère majeur pour obtenir une qualification au Conseil national des universités (CNU) et pour obtenir un poste. En matière de rémunération également, la publication d'articles est souvent associée à des primes, soit directement, comme c'est le cas dans les business schools qui attribuent des gratifications à chaque publication, soit de manière plus indirecte via une évaluation globale de la production, comme c'est le cas à l'université pour l'obtention de la Prime d'encadrement doctoral et de recherche (PEDR). Enfin, en matière d'évolution de carrière, la publication conditionne l'obtention de promotions, et notamment le passage du corps des maîtres de conférences (ou des professeurs assistants ou associés) à celui des professeurs.

Par-delà ces diverses gratifications, la production scientifique est également source de prestige au sein de la communauté scientifique - et même quelque fois au-delà -, au point que les chercheurs les plus reconnus sont parfois qualifiés de stars. L'appétit de connaissance peut alors se trouver converti en une soif de reconnaissance - Bourdieu parle ici d'un passage de la libido sciendi à la libido dominandi (Bourdieu, 1995). Par transitivité, les universités et les écoles de commerce voire les cabinets de conseil - qui emploient ces chercheurs acquièrent elles-mêmes un statut prestigieux, d'où la compétition actuelle pour attirer, motiver et fidéliser les plus reconnus d'entre nous.

Par ailleurs, outre la valorisation de leurs producteurs, les travaux scientifiques peuvent être euxmêmes valorisés en devenant des objets d'échange. Les cinquante dernières années ont vu l'apparition d'une industrie des connaissances en management considérable à laquelle appartiennent notamment les universités, les écoles de commerce, ou les cabinets de conseil, et qui génère annuellement des centaines de milliards d'euros (O’Mahoney, Heusinkveld et Wright, 2013 ; Pfeffer et Fong, 2004). Via leurs travaux, les chercheurs peuvent eux aussi capter une part 
de ces revenus, que ce soit par la publication d'ouvrages ou au travers de la réalisation d'activités de conseil - ce qu'ils font d'ailleurs de manière fréquente. En outre, en raison de leur expertise scientifique, ils bénéficient souvent d'une légitimité accrue, laquelle leur confère un avantage compétitif dans ce marché de la connaissance de plus en plus concurrentiel (Suddaby et Greenwood, 2001).

S'il est largement répandu, l'usage économique des connaissances produites par les sciences de gestion n'est pas sans faire peser des risques sur la qualité des travaux réalisés. Les deux critères habituels d'évaluation de la qualité des travaux scientifiques peuvent en effet se trouver affectés.

Il existe tout d'abord un risque d'affaissement de la rigueur scientifique. L'appât des divers gains qui peuvent être retirés des productions académiques a en effet pour conséquence un essor considérable du nombre de cas d'inconduite scientifique avérés (Gross, 2016). D'où une lutte croissante contre le plagiat et la fraude dans notre discipline comme dans le reste de la communauté scientifique. Il conduit aussi à la prolifération de travaux communiqués ou publiés dans des conférences ou dans des revues scientifiques aux standards d'évaluation médiocres lesquelles profitent de leur côté des fortes redevances qu'elles demandent aux chercheurs (SaintMartin, 2018). D'où l'utilité des divers classements de revues établis en France comme ailleurs dans le monde. Il conduit enfin à un morcellement des travaux en sous-champs relativement indépendants, qui ont pour principal but de permettre à leurs auteurs d'occuper une niche académique indépendamment de la cumulativité des connaissances produites (Mehrpouya et Willmott, 2018). D'où des appels à l'interdisciplinarité afin de contrer cette hyper-spécialisation.

Il existe également un risque d'affaiblissement de la pertinence des travaux qui sont menés. D'une part, la course à la publication dans les revues scientifiques les plus prestigieuses - la fameuse course aux étoiles - donne régulièrement lieu à la production d'articles extrêmement rigoureux mais aux implications managériales et sociales discutables. D'où des appels à ne pas relâcher nos traditionnelles exigences en matière de préconisations (Rouquet, 2017). D'autre part, la démarche de commodification des connaissances - d'élaboration des connaissances afin qu'elles acquièrent une valeur marchande - conduit souvent à proposer des cadres d'analyse simples qui pourront facilement être appréhendés par leurs récepteurs, au risque du simplisme (Suddaby et Greenwood, 2001). D'où, entre autres exemples, l'utilité d'initiatives telles que la 
labellisation d'ouvrages opérée par la FNEGE, qui vise précisément à s'assurer de la qualité des livres publiés dans notre champ.

\subsection{La fonction idéologique}

Si les trois fonctions précédemment évoquées sont bien connues, la fonction idéologique des connaissances scientifiques est quant à elle souvent passée sous silence. S'inscrivant dans la tradition positiviste, nombre de chercheurs considèrent en effet la production scientifique comme un système de propositions objectives portant sur le réel (Martinet, 2013). La science aurait pour but de dévoiler les lois qui gouvernent le monde et qui pré-existent aux observations; elle serait un discours produit indépendamment des caractéristiques de ses auteurs. Définir le travail scientifique en ces termes, c'est ignorer que la manière dont les chercheurs formulent les problèmes et y apportent des réponses est déterminée par leurs représentations ${ }^{6}$. Tout travail scientifique est assis sur un imaginaire, sur des postulats, le plus souvent implicites. Tout travail scientifique est à ce titre porteur d'idéologie - d'une vision particulière du monde -, et ceci pour deux raisons.

La recherche scientifique consiste tout d'abord à identifier des objets qui importent. L'infinité de phénomènes qui constituent le monde ne peut être examinée dans sa totalité, il faut donc déterminer ceux sur lesquels on porte le regard. Comme l'indique Bachelard, dans la vie scientifique, les problèmes ne se posent pas d'eux-mêmes. (...) Pour un esprit scientifique, toute connaissance est une réponse à une question. S'il n'y a pas eu de question, il ne peut y avoir connaissance scientifique. Rien ne va de soi. Rien n'est donné. Tout est construit » $(1938,14)$.

Historiquement, les sciences de gestion ont ainsi davantage examiné la performance économique plutôt que la performance sociale ou environnementale, les grandes entreprises plutôt que les petites structures, la place des hommes plutôt que celle des femmes, ou encore, les succès plutôt que les échecs. Ceci est bien entendu moins vrai à l'heure actuelle, où toute une série d'évolutions sociétales - l'essor des problématiques environnementales, l'engouement pour les

\footnotetext{
6 Bien entendu, l’idéologie du chercheur peut elle-même être dictée par les canons scientifiques de son époque. En son célèbre essai portant sur la structure des révolutions scientifiques, Kuhn montre que c’est précisément le rôle d'un paradigme scientifique dominant que de définir les problèmes et les méthodes légitimes d'un domaine de recherche (Kuhn, 1962).
} 
start-ups, la popularisation de la cause féminine, etc. - ont peu à peu conduit les chercheurs à s'intéresser à ces problématiques auparavant marginales. Si les préoccupations ont évolué, c'est bien entendu aussi en raison de l'apparition du courant des critical management studies qui, depuis les années 1990, a largement contribué à faire évoluer les thématiques traditionnellement abordées par les sciences de gestion (Adler, Forbes et Willmott, 2007).

Une fois les objets d'étude sélectionnés, la recherche scientifique consiste également à proposer des explications acceptables aux problèmes identifiés. Outre le fait qu'ils soient infinis, les phénomènes empiriques sont équivoques: différentes proposition théoriques peuvent être à même de rendre compte d'un même ensemble d'observations - à tout le moins temporairement. C'est la thèse de la sous-détermination des théories par l'expérience défendue par Quine (1970) et auparavant formulée en ces termes par Kuhn: "plusieurs constructions théoriques peuvent être échafaudées pour une collection de faits donnée » $(1962,113)$.

Au cours du temps, les sciences de gestion ont ainsi vu se succéder différentes inflexions dans leurs manières d'aborder ses objets d'étude. Entre autres exemples, notre discipline a ainsi d'abord envisagé le succès des entreprises à l'aune de leur environnement concurrentiel avant de considérer qu'il fallait également prendre en compte les ressources des organisations - avec ici le tournant de l'approche par les ressources inauguré par Edith Penrose (1959). Elle a aussi d'abord envisagé les contrats comme seuls régisseurs des relations économiques avant de considérer qu'ils devaient être complétés par de la confiance - avec ici le tournant de l'approche relationnelle dont les contributions séminales furent celles de Stewart Macaulay (1963) ou de Ian Macneil (2001). Elle a encore d'abord envisagé les acteurs économiques comme atomisés et opportunistes avant de mettre les effets réseaux et les liens sociaux au cœur de ses préoccupations - répondant ainsi à l'appel de l'examen de l'encastrement des activités économiques de Mark Granovetter (1985). Elle a enfin d'abord envisagé les pratiques avant de prendre en compte les discours - s'inscrivant de la sorte dans le linguistic turn impulsé par les post-structuralistes (Alvesson et Kärreman, 2000).

Pour les deux raisons que nous venons d'évoquer, immanquablement donc, le travail scientifique véhicule l'idéologie du chercheur qui le produit, c'est-à-dire la manière qu'il a d'appréhender le monde. Puisque toute production scientifique est nécessairement porteuse d'idéologie, ce qui fait 
ici problème, ce n'est pas tant le fait qu'une telle dimension idéologique existe, mais qu'elle soit régulièrement ignorée ou niée. Ignorée, car nombre de chercheurs persistent en effet encore à croire qu'ils ne sont que des témoins objectifs du fonctionnement du monde. Ou niée, avec pour motif évident d'avancer des idées particulières sous le sceau de l'objectivité la plus absolue. Comme le souligne Martinet : «la connaissance scientifique [est] inéluctablement construite et contingente, exprimant toujours le point de vue et le découpage discrétionnaire du chercheur et dépendante des schémas mentaux de celui-ci (...). La rhétorique est refoulée chez les positivistes dont la revendication de neutralité et d'objectivité de la connaissance produite est trop insistante pour ne pas être suspecte » $(2013,16)^{7}$.

\section{CONCLUSION}

L'objectif de cet article était de faire le point sur un sujet qui nous semble central pour tout chercheur, à savoir, l'identification des fonctions de ses travaux. Si la plupart de ces fonctions sont déjà bien connues, il s'agissait de les recenser de manière exhaustive, de montrer leur articulation, et de souligner la fonction idéologique, souvent passée sous silence. De notre point de vue, l'intérêt de ce travail à vocation didactique est double.

Il nous semble qu'un tel cadre d'analyse pourrait être utile pour préparer de futurs collègues à leurs activités de chercheurs ou pour informer toute personne intéressée par les enjeux de la recherche scientifique. En particulier, il permet de rappeler que toute production scientifique comporte nécessairement une dimension idéologique, et que la neutralité dont se prévalent souvent les chercheurs est en réalité une illusion. Ceci est d'importance en sciences de gestion, où les conséquences socio-économiques des travaux réalisés peuvent être particulièrement fortes.

Il nous semble que ce travail pourrait également être utile en matière de pilotage des activités de recherche. Il constitue d'une part une invitation à préciser les modalités d'évaluation de nos travaux. Souhaite-t-on nous en tenir aux enjeux épistémiques traditionnels, ou bien voulons-nous aussi porter certaines thématiques? Influencer les pratiques ? Défendre un bilan économique ? Il faudrait en conséquence revoir nos critères et dépasser les facteurs d'impact traditionnellement

Chacun reconnaîtra ici une critique largement adressée à l'endroit de la théorie des coûts de transaction (Ghoshal et Moran, 1996) ou de la théorie de l’agence (Boncori et Mahieux, 2012). 
employés pour inclure des indicateurs plus variés (pour un panorama des indicateurs possibles, voir Aguinis, Shapiro, Antonacopoulou et Cummings, 2014). Dans le même temps, ce travail permet d'autre part de rappeler les tensions associées à chacune des fonctions présentées. S'il peut être souhaitable d'inciter à un usage pratique ou économique des connaissances produites, ces usages ne doivent pas être les seuls à régir la conduite de nos travaux, au risque sinon de mettre en péril leur rigueur et leur pertinence scientifiques.

\section{BIBLIOGRAPHIE}

Acquier A. \& Aggeri F. (2015), Une généalogie de la pensée managériale sur la RSE, Revue française de gestion, vol. 41, $\mathrm{n}^{\circ} 253$, p. 387-413.

Adler P. S., Forbes L. C. \& Willmott H. (2007), Critical management studies, The academy of management annals, vol. 1, $\mathrm{n}^{\circ} 1$, p. 119-179.

Aggeri F. (2016), L’obsession de la productivité et la fabrique du chercheur publiant, Le Libellio d'Aegis, vol. 12, n 2, p. 21-32.

Aguinis H., Shapiro D. L., Antonacopoulou E. P. \& Cummings T. G. (2014), Scholarly impact: A pluralist conceptualization, Academy of Management Learning \& Education, vol. 13, $n^{\circ} 4$, p. 623-639.

Alvesson M. \& Kärreman D. (2000), Taking the linguistic turn in organizational research: Challenges, responses, consequences, The journal of applied behavioral science, vol. 36, $\mathrm{n}^{\circ} 2$, p. $136-158$.

Bachelard G. (1938), La formation de l'esprit scientifique, Paris, Vrin.

Bartunek J. M., Rynes S. L. \& Ireland R. D. (2006), What makes management research interesting, and why does it matter?, Academy of management Journal, vol. 49, $\mathrm{n}^{\circ}$ 1, p. 915.

Berchicci L. \& King A. (2007), Postcards from the edge: a review of the business and environment literature, The Academy of Management Annals, vol. 1, nº 1, p. 513-547.

Birkinshaw J., Hamel G. \& Mol M. J. (2008), Management innovation, Academy of management Review, vol. 33, n 4, p. 825-845.

Boncori A.-L. \& Mahieux X. (2012), Au-delà du bien et du mal, Revue française de gestion, vol. 9-10, n²28-229, p. 129-146. 
Bourdieu P. (1995), La cause de la science, Actes de la recherche en sciences sociales, vol. 106, $\mathrm{n}^{\circ}$ 1, p. 3-10.

Carton G. \& Mouricou P. (2017), A quoi sert la recherche en management? Une analyse systématique de la littérature anglo-saxonne sur le débat rigueur-pertinence (1994-2013), M@n@gement, vol.20,n², p. 166-203.

David A., Hatchuel A. \& Laufer R. (2012), Les nouvelles fondations des sciences de gestion: éléments d'épistémologie de la recherche en management, Presse des Mines.

Doty D. H. \& Glick W. H. (1994), Typologies as a unique form of theory building: Toward improved understanding and modeling, Academy of management review, vol. 19, $\mathrm{n}^{\circ} \mathrm{2}$, $\mathrm{p}$. 230-251.

Ghoshal S. \& Moran P. (1996), Bad for practice: A critique of the transaction cost theory, Academy of management Review, vol. 21, n 1, p. 13-47.

Gingras Y. (2018), Histoire des sciences, Presses universitaires de France.

Glaser B. G. \& Strauss A. L. (1967), Discovery of grounded theory: Strategies for qualitative research, Chicago, Aldine.

Granovetter M. (1985), Economic action and social structure: The problem of embeddedness, American journal of sociology, vol. 91, n 3, p. 481-510.

Gross C. (2016), Scientific misconduct, Annual review of psychology, vol. 67, p. 693-711.

Hatchuel A., Weil B. \& Le Masson P. (2013), Towards an ontology of design: lessons from C-K design theory and Forcing, Research in engineering design, vol. 24, n 2, p. 147-163.

Jacques-Chaquin N. \& Houdard S. (1998), Curiosité et Libido sciendi de la Renaissance aux Lumières : Théories, ENS éditions.

Kieser A. \& Leiner L. (2009), Why the rigour-relevance gap in management research is unbridgeable, Journal of Management Studies, vol. 46, n 3, p. 516-533.

Kieser A., Nicolai A. \& Seidl D. (2015), The practical relevance of management research: Turning the debate on relevance into a rigorous scientific research program, The Academy of Management Annals, vol. 9, $\mathrm{n}^{\circ}$ 1, p. 143-233.

Kuhn T. (1962), La structure des révolutions scientifiques, Flammarion.

Loewenstein G. (1994), The psychology of curiosity: A review and reinterpretation., Psychological bulletin, vol. 116, nº 1, p. 75.

Macaulay S. (1963), Non-contractual relations in business: A preliminary study, American Sociological Review, vol. 28, n 1, p. 55-67. 
Macneil I. R. (2001), The relational theory of contract: selected works of Ian MacNeil (Edited by D. Campbell), London: Sweet \& Maxwell.

Mandard M. (2019), Les usages organisationnels des espaces géographiques, Recherches en Sciences de Gestion, vol. 5, n 134 , p. 59-76.

Mandard M. (2020), Ce que l'on reproche aux sciences de gestion. Une synthèse des critiques adressées à notre discipline, 29ème conférence de l'Association internationale de management stratégique, Conférence en ligne.

Martinet A.-C. (2013), La genèse des sciences de gestion, Épistémologie des sciences de gestion, Paris, Vuibert, p. 3-20.

Mehrpouya A. \& Willmott H. (2018), Making a Niche: The Marketization of Management Research and the Rise of 'Knowledge Branding', Journal of Management Studies, vol. 55, $\mathrm{n}^{\circ} 4$, p. 728-734.

Moriceau J.-L., Laroche H. \& Jardat R. (2017), Recherche en gestion, le sens mis en pièces, Revue française de gestion, vol. 6, n 267, p. 67-78.

O’Mahoney J., Heusinkveld S. \& Wright C. (2013), Commodifying the commodifiers: The impact of procurement on management knowledge, Journal of Management Studies, vol. 50, n², p. 204-235.

Penrose E. T. (1959), The theory of the growth ofthe firm, New York: Sharpe.

Pfeffer J. (2007), A modest proposal: How we might change the process and product of managerial research, Academy of Management journal, vol. 50, n 6, p. 1334-1345.

Pfeffer J. \& Fong C. T. (2004), The business school 'business': Some lessons from the US experience, Journal of management studies, vol. 41, nº 8, p. 1501-1520.

Pichault F. (2013), Gestion du changement. Vers un management polyphonique, De Boeck.

Pichault F., Lisein O., Rondeaux G. \& Xhauflair V. (2008), La recherche-intervention peut-elle être socialement responsable ?, Paris, Vuibert.

Quine W. V. (1970), On the reasons for indeterminacy of translation, The Journal of Philosophy, vol. $67, n^{\circ} 6$, p. 178-183.

Rouquet A. (2017), Face à la tyrannie des étoiles: révoltons-nous!, Revue française de gestion, vol. , $\mathrm{n}^{\circ} 6$, p. 133-147.

Ryan R. M. \& Deci E. L. (2000), Self-determination theory and the facilitation of intrinsic motivation, social development, and well-being., American psychologist, vol. 55, $\mathrm{n}^{\circ} 1$, p. 68. 
Saint-Martin A. (2018), L'édition scientifique «piratée». Passage en revue et esquisse de problématisation, Zilsel, vol. , n², p. 179-202.

Savall H. \& Zardet V. (2014), Reconstruire l'entreprise: Les fondements du management socioéconomique, Dunod.

Simon H. A. (2001), “Seek and Ye Shall Find”: How Curiosity Engenders Discovery, Designing for science, Psychology Press, p. 19-32.

Suddaby R. et Greenwood R. (2001), Colonizing knowledge: Commodification as a dynamic of jurisdictional expansion in professional service firms, Human relations, vol. 54, n 7, p. 933-953.

Verstraete T. (2007), A la recherche des sciences de gestion, Revue française de gestion, vol. 9-10, n 178-179, p. 91-105. 